\title{
Density functional theory and quantum computation
}

\author{
Frank Gaitan ${ }^{1,2,3}$ and Franco Nori ${ }^{1,3,4}$ \\ ${ }^{1}$ Advanced Science Institute, The Institute of Physical and Chemical Research (RIKEN), \\ Wako-shi, Saitama 351-0198, Japan \\ ${ }^{2}$ Department of Physics, Southern Illinois University, Carbondale, IL 62901-4401 \\ ${ }^{3}$ CREST, Japan Science and Technology Agency (JST), \\ Kawaguchi, Saitama, 332-0012, Japan \\ ${ }_{4}^{4}$ Physics Department, Center for Theoretical Physics, \\ University of Michigan, Ann Arbor, MI 48109
}

(Dated: October 22, 2018)

\begin{abstract}
This paper establishes the applicability of density functional theory methods to quantum computing systems. We show that ground-state and time-dependent density functional theory can be applied to quantum computing systems by proving the Hohenberg-Kohn and Runge-Gross theorems for a fermionic representation of an $N$ qubit system. As a first demonstration of this approach, time-dependent density functional theory is used to determine the minimum energy gap $\Delta(N)$ arising when the quantum adiabatic evolution algorithm is used to solve instances of the NP-Complete problem MAXCUT. It is known that the computational efficiency of this algorithm is largely determined by the large- $N$ scaling behavior of $\Delta(N)$, and so determining this behavior is of fundamental significance. As density functional theory has been used to study quantum systems with $N \sim 10^{3}$ interacting degrees of freedom, the approach introduced in this paper raises the realistic prospect of evaluating the gap $\Delta(N)$ for systems with $N \sim 10^{3}$ qubits. Although the calculation of $\Delta(N)$ serves to illustrate how density functional theory methods can be applied to problems in quantum computing, the approach has a much broader range, and shows promise as a means for determining the properties of very large quantum computing systems.
\end{abstract}

PACS numbers: 31.15.ee,03.67.Ac 


\section{INTRODUCTION}

The inability of a classical computer to efficiently simulate the dynamics of a quantum system is well-known. The problem is that the dimension of the Hilbert space grows exponentially with the number of degrees of freedom of the quantum system, and this in turn causes an exponential growth in the amount of memory and CPU-time required to carry out the simulation. This inefficiency is a major stumbling block for numerical studies aiming to determine the asymptotic performance of quantum algorithms. For example, numerical simulation of the dynamics of the quantum adiabatic evolution (QAE) algorithm applied to the NP-Complete problem Exact Cover 3 has been limited to systems containing $N \leq 20$ qubits 1,2 . Because the algorithm dynamics must be adiabatic, its runtime $T$ must satisfy the inequality

$$
T \gg \frac{M}{\Delta^{2}}
$$

where

$$
\begin{aligned}
M & =\max _{0 \leq s \leq 1}\left|\left\langle E_{1}(s)\left|\frac{d H(s)}{d s}\right| E_{0}(s)\right\rangle\right| ; \\
\Delta & =\min _{0 \leq s \leq 1}\left[E_{1}(s)-E_{0}(s)\right] ;
\end{aligned}
$$

and here $t$ is time; $s=t / T$ is dimensionless time; $H(s)$ is the time-dependent Hamiltonian that drives the dynamics of the QAE algorithm; and $\left\{E_{i}(s),\left|E_{i}(s)\right\rangle: i=0, \ldots, 2^{N}-1\right\}$ are the eigenvalues and eigenstates of $H(s)$. In the usual formulation ${ }^{3,4}, \underline{5}$ of $\mathrm{QAE}, d H(s) / d s$ is an $s$-independent matrix whose largest eigenvalue bounds $M$. Typically, this eigenvalue scales polynomially with $N$. Thus, if the minimum gap $\Delta(N)$ separating the ground- and first-excited states scales polynomially (exponentially) with $N$, so will the algorithm runtime $T(N)$. An efficient (inefficient) algorithm ${ }^{6}$ for a computational problem is one that solves all instances of the problem with polynomial (exponential) $T(N)$. We see then that the computational efficiency of the QAE algorithm is largely determined by the scaling behavior of the minimum gap $\Delta(N)$. Attempts to evaluate $\Delta(N)$ using exact diagonalization ${ }^{7}$ have been limited to $N \leq 20$ qubits. Recently, however, the minimum gap $\Delta(N)$ for QAE applied to Exact Cover 3 has been determined for $N \leq 128$ qubits using quantum Monte Carlo methods ${ }^{8}$. This represents a substantial technical advance, and has stirred great interest in finding other computational approaches that might allow quantum algorithm performance 
to be determined for still larger qubit systems.

Quantum computation is not the only research area struggling with the difficulties of simulating quantum systems $\stackrel{9}{ }$. Condensed-matter physicists and quantum chemists have been working under the shadow of this problem for decades. A number of computational approaches have been developed which, together with increasingly more powerful computers, have allowed much progress to be made, in spite of the ultimately unavoidable difficulties involved. Among these approaches, density functional theory (DFT) has proven to be one of the most successful $\underline{10}, \underline{12}$. DFT is a theory of interacting fermion systems. It provides an exact treatment of all many-body effects through the exchange-correlation energy functional. It can also handle the coupling of such fermion systems to both static and time-varying electric and magnetic fields. Ground-state density functional theory (GS-DFT) has been used to determine a wide range of ground-state properties of atomic, molecular, and solid state systems ${ }^{13}, \frac{14}{4}$; while time-dependent density functional theory (TD-DFT) has been used to determine excited-state properties, as well as the linear and non-linear response of interacting many-electron systems to electromagnetic fields 15,16 . For our purposes, it is especially significant that DFT has been successfully applied to quantum systems containing $N \sim 10^{3}$ interacting degrees of freedom $\underline{17, \underline{18}, 19}$.

In this paper we establish the applicability of DFT methods to quantum computing systems. By establishing this link, we shall see that a powerful tool becomes available for determining the properties of very large quantum computing systems. Although our analysis can be extended to the case of $N$ qudits ( $d$-level systems) residing on a $D$-dimensional lattice, we restrict the presentation to $N$ qubits residing on a $2 \mathrm{D}$ lattice since this corresponds to the experimentally interesting cases of qubits placed in a $2 \mathrm{D}$ ion $\operatorname{trap}^{20}$, or restricted to a planar superconducting qubit circuit $^{21}$.

The outline of this paper is as follows. We begin in Section $\amalg$ by showing how an $N$ qubit system can be transformed into a system of $N$ lattice fermions, and then in Section III, illustrate this transformation by using it to re-write the dynamics of the QAE algorithm applied to the NP-Complete problem $M A X C U T^{22}$. For the resulting interacting fermion system, Section IV establishes the Hohenberg-Kohn 13 and Runge-Gross ${ }^{15}$ theorems, and sets up the auxiliary Kohn-Sham system of non-interacting fermions $\stackrel{14}{ }$. The results of Section IV provide the justification for applying GS- and TD-DFT to quantum computing systems. The proofs given in Section [V] are adaptations of well-established proofs used for interacting electron 
systems, and so their validity should not be in doubt. Section $\mathrm{V}$ works out the linear response of the system of interacting fermionized qubits using TD-DFT, and as an application, shows how this response can be used to determine the minimum energy gap $\Delta(N)$ for the MAXCUT dynamics. Here we begin to see the value of the newly established link between DFT and quantum computing. Calculation of $\Delta(N)$ boils down to a calculation of excitation energies, and the reliable calculation of excitation energies for very large interacting electron systems was one of the first triumphs of TD-DFT. A straightforward adaptation of standard TD-DFT arguments then determines $\Delta(N)$. In light of earlier remarks, the link established in this paper between DFT and quantum computing raises the realistic prospect of evaluating the minimum gap $\Delta(N)$ for $N \sim 10^{3}$ qubits, and thus of studying the performance of the QAE algorithm for much larger qubit systems than is currently possible using other approaches. Although we focus on the calculation of the minimum gap in this paper, it is clear that the application of DFT to quantum computing systems has a much broader range, and shows genuine promise as a means for determining the properties of very large quantum computing systems. Finally, the paper closes in Section VI with a discussion of future work.

\section{QUBIT-FERMION TRANSFORMATION}

Consider $N$ qubits residing on an $N$-site 2D lattice with basis vectors $\hat{\mathbf{e}}_{k}(k=1,2)$, and sites specified by the position vector $\mathbf{r}$. Let $\boldsymbol{\sigma}(\mathbf{r})$ denote the Pauli matrices associated with the qubit at $\mathbf{r}$. We now show how the qubits can be converted into lattice fermions via the 2D Jordan-Wigner (JW) transformation ${ }^{23}$. Note that the following analysis can be extended to $N$ qudits (viz. $N d$-level systems) on a $D$-dimensional lattice using the generalized JW transformation $\underline{24}$ that fermionizes a spin $s$ system $(d=2 s+1)$ in $D$ spatial dimensions.

For a $2 \mathrm{D}$ system of qubits, the JW transformation is:

$$
\begin{aligned}
\sigma^{+}(\mathbf{r}) & =2 a_{\mathbf{r}}^{\dagger} Q_{\mathbf{r}} \\
\sigma^{-}(\mathbf{r}) & =2 Q_{\mathbf{r}}^{\dagger} a_{\mathbf{r}} \\
\sigma_{z}(\mathbf{r}) & =2 \hat{n}_{\mathbf{r}}-1 .
\end{aligned}
$$

Here: $\sigma^{ \pm}(\mathbf{r})=\sigma_{x}(\mathbf{r}) \pm i \sigma_{y}(\mathbf{r}) ; a_{\mathbf{r}}^{\dagger}\left(a_{\mathbf{r}}\right)$ creates (annihilates) a lattice fermion at $\mathbf{r} ; \hat{n}_{\mathbf{r}}=a_{\mathbf{r}}^{\dagger} a_{\mathbf{r}}$ 
is the fermion number operator at $\mathbf{r}$; and

$$
Q_{\mathbf{r}}=\exp \left[-i \phi_{\mathbf{r}}\right] ; \quad \phi_{\mathbf{r}}=\left(\frac{1}{2 \pi \theta}\right) \sum_{\mathbf{r}^{\prime}} \Phi\left(\mathbf{r}, \mathbf{r}^{\prime}\right) \hat{n}_{\mathbf{r}^{\prime}}
$$

In Eq. (4), $\Phi\left(\mathbf{r}, \mathbf{r}^{\prime}\right)$ is the angle made by $\left(\mathbf{r}-\mathbf{r}^{\prime}\right)$ with respect to some reference direction, say $\hat{\mathbf{e}}_{1}$. Thus: (i) $\Phi\left(\mathbf{r}, \mathbf{r}^{\prime}\right)$ changes by $2 \pi$ when $\left(\mathbf{r}-\mathbf{r}^{\prime}\right)$ traces out a closed loop around $\mathbf{r}^{\prime}$; and (ii) by convention, $\Phi(\mathbf{r}, \mathbf{r}) \equiv 0$. The requirement that the Pauli operators $\boldsymbol{\sigma}(\mathbf{r})$ commute at different lattice sites forces $\theta$ to satisfy

$$
\frac{1}{2 \pi \theta}=2 m+1 \quad(m=0, \pm 1, \pm 2, \cdots)
$$

in Eq. (4).

As shown in Ref. 23, the lattice fermions are spinless, and minimally-coupled to a gauge field $A_{k}(\mathbf{r})=\Delta_{k} \phi_{\mathbf{r}} \equiv \phi_{\mathbf{r}+\hat{\mathbf{e}}_{k}}-\phi_{\mathbf{r}}$. The action for the gauge-field $A_{\mu}(\mathbf{r})$ is given by the Chern-Simons term 25

$$
\mathcal{A}_{c s}=-\frac{\theta}{4} \int d t \sum_{\mathbf{r}} \epsilon^{\mu \nu \lambda} A_{\mu}(\mathbf{r}) F_{\nu \lambda}(\mathbf{r}) .
$$

Maxwell's equations for this system take the form

$$
j_{\mathbf{r}}^{\mu}=\epsilon^{\mu \nu \lambda} F_{\nu \lambda}(\mathbf{r}),
$$

where $j_{\mathbf{r}}^{\mu}$ is the fermion current, $F_{\nu \lambda}(\mathbf{r})$ is the gauge field tensor, $\epsilon^{\mu \nu \lambda}$ is the totally antisymmetric Levi-Civita tensor, and $\mu, \nu, \lambda=0,1,2$. From Eq. (5), the fermion current $j_{\mathbf{r}, \mu}$ has components

$$
\begin{aligned}
& j_{\mathbf{r}, 0}=n_{\mathbf{r}}, \\
& j_{\mathbf{r}, k}=\frac{1}{2 \pi} \sum_{\mathbf{y}}\left\{\Delta_{k} G_{\mathbf{r}, \mathbf{y}}\right\}\left\{\partial_{t} n_{\mathbf{y}}\right\} \quad(k=1,2),
\end{aligned}
$$

where $G_{\mathbf{r}, \mathbf{y}}$ is the Green's function for the lattice Laplacian

$$
\sum_{k=1,2} \Delta_{k} \Delta_{k} G_{\mathbf{r}, \mathbf{y}}=-2 \pi \delta_{\mathbf{r}, \mathbf{y}} .
$$

Fermion current conservation, $\partial_{\mu} j^{\mu}=0$, follows immediately from Maxwell's equations.

\section{APPLICATION: NP-COMPLETE PROBLEM MAXCUT}

In the problem MAXCUT, one considers an $N$-node undirected graph $\mathcal{G}$ with nodes specified by $\mathbf{r}$. The nodes (edges) are assigned weights $w_{\mathbf{r}}\left(w_{\mathbf{r}, \mathbf{r}^{\prime}}\right)$, and a binary variable $s_{\mathbf{r}}$ 
is associated with each node $\mathbf{r}$. A cut of the graph $\mathcal{G}$ is a partition of the nodes into two sets $\mathcal{S}_{0}$ and $\mathcal{S}_{1}$. For all nodes belonging to $\mathcal{S}_{0}\left(\mathcal{S}_{1}\right), s_{\mathbf{r}}$ is assigned the value 0 (1). The node variables are used to construct a string variable $s=s_{\mathbf{r}_{1}} \cdots s_{\mathbf{r}_{N}}$, and all possible assignments of the $N$ (binary) node variables leads to $2^{N}$ possible string assignments for $s$. It follows that there is a one-to-one correspondence between cuts of $\mathcal{G}$ and string assignments for $s$. The MAXCUT problem is to find the cut (viz. string assignment) that maximizes the payoff function $P(s)$ given by

$$
P(s)=\sum_{\mathbf{r}} s_{\mathbf{r}} w_{\mathbf{r}}+\sum_{\mathbf{r}, \mathbf{r}^{\prime}} s_{\mathbf{r}}\left(1-s_{\mathbf{r}^{\prime}}\right) w_{\mathbf{r}, \mathbf{r}^{\prime}}
$$

MAXCUT is known $\underline{6}$ to be NP-Complete, and so it belongs to the set of "hardest problems" in the complexity class NP.

The QAE algorithm was applied to MAXCUT in Ref. 22, where the dynamics is driven by the Hamiltonian

$$
H(t)=\left(1-\frac{t}{T}\right) H_{0}+\left(\frac{t}{T}\right) H_{P}
$$

Here $T$ is the algorithm runtime,

$$
H_{0}=\sum_{\mathbf{r}} \sigma_{x}(\mathbf{r})
$$

and

$$
\begin{aligned}
H_{P}=\sum_{\mathbf{r}} w_{\mathbf{r}}(1 & \left.-\sigma_{z}(\mathbf{r})\right) / 2 \\
& +\sum_{\mathbf{r}, \mathbf{r}^{\prime}} w_{\mathbf{r}, \mathbf{r}^{\prime}}\left(1-\sigma_{z}(\mathbf{r}) \sigma_{z}\left(\mathbf{r}^{\prime}\right)\right) / 2 .
\end{aligned}
$$

The Hamiltonian $H_{P}$ is known as the problem Hamiltonian. From Eq. (9), its eigenstates are the simultaneous eigenstates of the $\left\{\sigma_{z}(\mathbf{r})\right\}$ :

$$
\sigma_{z}(\mathbf{r})\left|s_{\mathbf{r}_{1}} \cdots s_{\mathbf{r}_{N}}\right\rangle=(-1)^{s \mathbf{r}}\left|s_{\mathbf{r}_{1}} \cdots s_{\mathbf{r}_{N}}\right\rangle
$$

By construction ${ }^{1,22}$, each bit string $s=s_{1} \cdots s_{N}$ that maximizes the MAXCUT payoff function labels a ground-state $\left|s_{1} \cdots s_{N}\right\rangle$ of $H_{P}$. The QAE algorithm places the qubit system in the ground-state of the initial Hamiltonian $H_{0}$, and for runtime $T$ sufficiently large, $H(t)$ evolves the quantum state adiabatically so that at time $T$, the system is in the ground-state of the final Hamiltonian $H_{P}$ with probability approaching 1 . Measurement of the $\left\{\sigma_{z}(\mathbf{r})\right\}$ at time $T$ yields, with probability approaching 1 , a string $s_{1} \cdots s_{N}$ that solves the MAXCUT instance. 
Using Eqs. (3) in $H(t)$ gives the fermionized QAE Hamiltonian for MAXCUT:

$$
\begin{aligned}
H(t)= & \left(1-\frac{t}{T}\right) \sum_{\mathbf{r}}\left[a_{\mathbf{r}}^{\dagger} Q_{\mathbf{r}}+Q_{\mathbf{r}}^{\dagger} a_{\mathbf{r}}\right] \\
& \left.+\left(\frac{t}{T}\right) \sum_{\mathbf{r}} v_{\mathbf{r}} \hat{n}_{\mathbf{r}}+\left(\frac{t}{T}\right) \sum_{\mathbf{r}} \sum_{\mathbf{r}^{\prime} \neq \mathbf{r}} w_{\mathbf{r}, \mathbf{r}^{\prime}} \hat{n}_{\mathbf{r}} \hat{n}_{\mathbf{r}^{\prime}}\right]
\end{aligned}
$$

where

$$
\begin{aligned}
w_{\mathbf{r}} & \equiv v_{\mathbf{r}}+W_{\mathbf{r}} ; \\
W_{\mathbf{r}} & =\sum_{\mathbf{r}^{\prime} \neq \mathbf{r}} w_{\mathbf{r}, \mathbf{r}^{\prime}} ;
\end{aligned}
$$

and a term proportional to the identity has been suppressed.

\section{DENSITY FUNCTIONAL THEORY}

In this Section we establish the applicability of the Hohenberg-Kohn and Runge-Gross theorems to the QAE/MAXCUT problem. These theorems justify the use of, respectively, ground-state and time-dependent density functional theory to the MAXCUT dynamics. Throughout, the ground-state is assumed to be non-degenerate, as would be appropriate for a non-vanishing minimum gap $\Delta$. The formalism can be extended, however, to cover degenerate ground-states $\underline{26,27}$.

\section{A. Ground-State Density Functional Theory}

We have seen that the QAE algorithm has an adiabatic dynamics that is driven by a slowly-varying Hamiltonian $H(t)$. In this subsection we focus on the MAXCUT Hamiltonian $H(t)$ at a fixed instant of time $t=t_{*}$. By fixing the time, we obtain a well-defined static Hermitian operator $H_{*} \equiv H\left(t=t_{*}\right)$. The aim of this subsection is to show that the Hohenberg-Kohn theorem applies to $H_{*}$. With this theorem in place, GS-DFT can be used to study the ground-state properties of $H_{*}=H\left(t=t_{*}\right)$ for any specific intermediate time $0<t_{*}<T$. We stress that even though the QAE algorithm works with a slowly-varying Hamiltonian, the discussion in this subsection is restricted to the static Hermitian operator $H_{*}=H\left(t_{*}\right)$ that is the value of $H(t)$ at the time $t=t_{*}$. 
Our starting point is the energy functional for the instantaneous MAXCUT Hamiltonian $H_{*} \equiv H\left(t=t_{*}\right)$ :

$$
E[n]=\min _{|\psi\rangle \rightarrow n}\left\langle\psi\left|H_{*}\right| \psi\right\rangle
$$

The domain of $E[n]$ is the set of all $N$-representable site occupation functions (SOF) $n_{\mathbf{r}}$ that can be obtained from an $N$-fermion wavefunction. The minimization in Eq. (11) is over all $|\psi\rangle$ for which

$$
n \equiv n_{\mathbf{r}}=\left\langle\psi\left|\hat{n}_{\mathbf{r}}\right| \psi\right\rangle
$$

and the minimizing state $\left|\psi_{\min }[n]\right\rangle$ is thus a functional of $n_{\mathbf{r}}$. Let $\left|\psi^{g}\right\rangle$ denote the groundstate of $H_{*} ; E^{g}$ the ground-state energy; and $n_{\mathbf{r}}^{g}$ the ground-state SOF. Inserting Eq. (10) into (11) gives

$$
E[n]=\left(t_{*} / T\right) \sum_{\mathbf{r}} v_{\mathbf{r}} n_{\mathbf{r}}+Q[n]
$$

where

$$
Q[n] \equiv \min _{|\psi\rangle \rightarrow n}\left\langle\psi\left|\left(T_{t_{*}}+U_{t_{*}}\right)\right| \psi\right\rangle,
$$

and $T_{t_{*}}$ and $U_{t_{*}}$ are the first and third terms, respectively, on the RHS of Eq. (10) at $t=t_{*}$.

To establish the Hohenberg-Kohn $(\mathrm{HK})$ theorem for $H_{*}$ we must show $\underline{28}$ : (i) $E\left[n^{g}\right]=E^{g}$; (ii) for $n_{\mathbf{r}} \neq n_{\mathbf{r}}^{g}, E[n]>E^{g}$; and (iii) the ground-state expectation value of any observable is a unique functional of the ground-state $\operatorname{SOF} n_{\mathbf{r}}^{g}$. By the variational principle, $\left\langle\psi\left|H_{*}\right| \psi\right\rangle \geq E^{g}$, with equality when $|\psi\rangle=\left|\psi^{g}\right\rangle$. Thus, for $n=n^{g}$, the search in Eq. (11) returns the ground-state $\left|\psi^{g}\right\rangle$ as the state $\left|\psi_{\min }\left[n^{g}\right]\right\rangle$ that minimizes $E\left[n^{g}\right]$. It follows that

$$
E\left[n^{g}\right]=\left\langle\psi^{g}\left|H_{*}\right| \psi^{g}\right\rangle=E^{g} .
$$

This establishes condition (i). For $n \neq n^{g}$, the minimizing state $\left|\psi_{\min }[n]\right\rangle \neq\left|\psi^{g}\left[n^{g}\right]\right\rangle$, and so by the variational principle,

$$
E[n]=\left\langle\psi_{\min }[n]\left|H_{*}\right| \psi_{\min }[n]\right\rangle>E^{g}
$$

This establishes condition (ii). Finally, since the ground-state $\left|\psi^{g}\right\rangle=\left|\psi_{\min }\left[n^{g}\right]\right\rangle$, it is a functional of $n^{g}$, and consequently, so are all ground-state expectation values:

$$
\begin{aligned}
\langle\hat{\mathcal{O}}\rangle_{g s} & =\left\langle\psi^{g}|\hat{\mathcal{O}}| \psi^{g}\right\rangle \\
& =\left\langle\psi_{\min }\left[n^{g}\right]|\hat{\mathcal{O}}| \psi_{\min }\left[n^{g}\right]\right\rangle \\
& =\mathcal{O}\left[n^{g}\right] .
\end{aligned}
$$


Condition (iii) is thus established, completing the proof of the HK theorem for $H_{*}=H\left(t_{*}\right)$.

To obtain a practical calculational scheme, an auxiliary system of non-interacting KohnSham (KS) fermions is introduced $\stackrel{14}{ }$, and it is assumed that the ground-state SOF $n_{\mathbf{r}}^{g}$ can be obtained from the ground-state density of the KS fermions moving in an external potential $v_{\mathbf{r}}^{k s}$. For $H_{*}=H\left(t_{*}\right)$, the KS Hamiltonian $H_{k s}=T_{t_{*}}^{\prime}+V^{k s}$ is defined to be:

$$
H_{k s}=\sum_{\mathbf{r}}\left(1-\frac{t_{*}}{T}\right)\left\{q_{\mathbf{r}} a_{\mathbf{r}}^{\dagger}+q_{\mathbf{r}}^{*} a_{\mathbf{r}}\right\}+\sum_{\mathbf{r}}\left(\frac{t_{*}}{T}\right) v_{\mathbf{r}}^{k s} \hat{n}_{\mathbf{r}},
$$

where $q_{\mathbf{r}}=\left\langle Q_{\mathbf{r}}\right\rangle$ is the ground-state expectation value of $Q_{\mathbf{r}}$. The effects of $Q_{\mathbf{r}}$ are thus incorporated into the KS dynamics through the mean-field $q_{\mathrm{r}}$. The KS energy functional $\epsilon_{k s}[n]$ is:

$$
\epsilon_{k s}[n]=\min _{|\psi\rangle \rightarrow n}\left\langle\psi\left|H_{k s}\right| \psi\right\rangle=T_{t_{*}}^{\prime}[n]+\sum_{\mathbf{r}}\left(\frac{t_{*}}{T}\right) v_{\mathbf{r}}^{k s} n_{\mathbf{r}} .
$$

To determine the KS external potential $v_{\mathbf{r}}^{k s}$, we re-write Eq. (11) as

$$
E[n]=T_{t_{*}}^{\prime}[n]+\sum_{\mathbf{r}}\left(\frac{t_{*}}{T}\right) v_{\mathbf{r}} n_{\mathbf{r}}+\xi_{x c}[n],
$$

where

$$
\xi_{x c}[n] \equiv Q[n]-T_{t_{*}}^{\prime}[n]
$$

is the exchange-correlation energy functional. As noted in Section I, it is through the exchange-correlation energy functional $\xi_{x c}[n]$ that DFT accounts for all many-body effects. Since $n_{\mathrm{r}}^{g}$ minimizes both $\epsilon_{k s}[n]$ and $E[n]$, Eqs. (12) and (13) are stationary about $n=n^{g}$. Taking their functional derivatives with respect to $n$, evaluating the result at $n=n^{g}$, and eliminating $\delta T_{t_{*}}^{\prime} /\left.\delta n\right|_{n=n^{g}}$ gives

$$
v_{\mathbf{r}}^{k s}=v_{\mathbf{r}}+\left(\frac{T}{t_{*}}\right) v_{x c}\left[n^{g}\right](\mathbf{r}),
$$

for $t_{*} \neq 0$. Here $v_{x c}\left[n^{g}\right](\mathbf{r})$ is the exchange-correlation potential which is the functional derivative of the exchange-correlation energy functional $\xi_{x c}\left[n^{g}\right]$ :

$$
v_{x c}\left[n^{g}\right](\mathbf{r})=\frac{\delta \xi_{x c}\left[n^{g}\right]}{\delta n_{\mathbf{r}}^{g}} .
$$

This sets in place the formulas for a self-consistent calculation of the ground-state properties of $H_{*}=H\left(t_{*}\right)$ using GS-DFT. Entanglement $\underline{29}$ and its links to quantum phase transitions $\underline{30}$ have been studied using GS-DFT. 


\section{B. Time Dependent Density Functional Theory}

Here we establish the Runge-Gross theorem ${ }^{15}$ for the instantaneous MAXCUT dynamics. Thus we focus on the instantaneous Hamiltonian $H_{*}=H\left(t_{*}\right)$ for a fixed $t_{*}\left(0<t_{*}<T\right)$. Now, however, we suppose that the external potential $v_{\mathbf{r}}$ in $H\left(t_{*}\right)$ begins to vary at a moment we call $t=0$. For $t \leq 0, v_{\mathbf{r}}(t)=v_{\mathbf{r}}$, and the fermions are in the ground-state $\left|\psi_{0}\right\rangle$ of $H\left(t_{*}\right)$. The Runge-Gross theorem states that the SOFs $n_{\mathbf{r}}(t)$ and $n_{\mathbf{r}}^{\prime}(t)$ evolving

from a common initial state $|\psi(0)\rangle=\left|\psi_{0}\right\rangle$ under the influence of the respective potentials $V_{\mathbf{r}}(t)$ and $V_{\mathbf{r}}^{\prime}(t)$ (both Taylor-series expandable about $t=0$ ) will be different provided that $\left[V_{\mathbf{r}}(t)-V_{\mathbf{r}}^{\prime}(t)\right] \neq C(t)$. For us:

$$
\begin{aligned}
V_{\mathbf{r}}(t) & =\left(\frac{t_{*}}{T}\right)\left(1-\frac{t_{*}}{T}\right) v_{\mathbf{r}}(t) \\
V_{\mathbf{r}}^{\prime}(t) & =\left(\frac{t_{*}}{T}\right)\left(1-\frac{t_{*}}{T}\right) v_{\mathbf{r}}^{\prime}(t)
\end{aligned}
$$

and

$$
\begin{aligned}
V_{\mathbf{r}}(t) & =\sum_{k=0}^{\infty} a_{k}(\mathbf{r}) t^{k} / k ! \\
V_{\mathbf{r}}^{\prime}(t) & =\sum_{k=0}^{\infty} a_{k}^{\prime}(\mathbf{r}) t^{k} / k ! .
\end{aligned}
$$

Let $C_{k}(\mathbf{r}) \equiv a_{k}(\mathbf{r})-a_{k}^{\prime}(\mathbf{r})$. The condition that $\left[V_{\mathbf{r}}(t)-V_{\mathbf{r}}^{\prime}(t)\right] \neq C(t)$ means a smallest integer $K$ exists such that $C_{k}(\mathbf{r})$ is a non-trivial function of $\mathbf{r}$ for all $k \geq K$, while for $k<K$, it is a constant $C_{k}$ which can be set to zero without loss of generality.

Recall (Eq. (6) ) that the conserved fermion current has components

$$
\begin{aligned}
& \hat{j}_{\mathbf{r}, 0}(t)=n_{\mathbf{r}}(t) \\
& \hat{j}_{\mathbf{r}, k}(t)=\left(\frac{1}{2 \pi}\right) \sum_{\mathbf{y}}\left(\Delta_{k} G_{\mathbf{r}, \mathbf{y}}\right) \partial_{t} n_{\mathbf{y}}(t),
\end{aligned}
$$

with $k=1,2$. Defining $j_{\mathbf{r}, k}(t)=\left\langle\psi_{0}\left|\hat{j}_{\mathbf{r}, k}(t)\right| \psi_{0}\right\rangle$, it follows that

$$
\partial_{t}\left\{j_{\mathbf{r}, k}(t)-j_{\mathbf{r}, k}^{\prime}(t)\right\}=\left\langle\psi_{0}\left|\left[\hat{j}_{\mathbf{r}, k}(t), H(t)-H^{\prime}(t)\right]\right| \psi_{0}\right\rangle
$$

Here $j_{\mathbf{r}, k}(t)\left(j_{\mathbf{r}, k}^{\prime}(t)\right)$ and $H(t)\left(H^{\prime}(t)\right)$ are the expected fermion current and the Hamiltonian, respectively, when the external potential is $v_{\mathbf{r}}(t)\left(v_{\mathbf{r}}^{\prime}(t)\right)$. The Hamiltonians $H(t)$ and $H^{\prime}(t)$ differ only in the external potential. Defining

$$
\delta j_{\mathbf{r}, k}(t)=j_{\mathbf{r}, k}(t)-j_{\mathbf{r}, k}^{\prime}(t)
$$


and

$$
\delta V_{\mathbf{y}}(t)=V_{\mathbf{y}}(t)-V_{\mathbf{y}}^{\prime}(t),
$$

evaluation of the commutator in Eq. (15) eventually gives

$$
\partial_{t}\left\{\delta j_{\mathbf{r}, k}(t)\right\}=-\left(\frac{1}{2 \pi}\right) \sum_{\mathbf{y}}\left(\Delta_{k} G_{\mathbf{r}, \mathbf{y}}\right) \delta V_{\mathbf{y}}(t) \mathcal{M}_{\mathbf{y}}(t),
$$

where

$$
\mathcal{M}_{\mathbf{y}}(t)=\left\langle\psi_{0}\left|\left(a_{\mathbf{y}}^{\dagger} Q_{\mathbf{y}}+Q_{\mathbf{y}}^{\dagger} a_{\mathbf{y}}\right)\right| \psi_{0}\right\rangle .
$$

With $K$ defined as above, taking $K$ time-derivatives of Eq. (16) and evaluating the result at $t=0$ gives

$$
\left.\frac{\partial^{K+1}}{\partial t^{K+1}}\left(\delta j_{\mathbf{r}, k}(t)\right)\right|_{0}=-\left(\frac{1}{2 \pi}\right) \sum_{\mathbf{y}}\left(\Delta_{k} G_{\mathbf{r}, \mathbf{y}}\right) \mathcal{M}_{\mathbf{y}}(0) C_{K}(\mathbf{r}),
$$

where we have used that

$$
\left.\frac{\partial^{k}}{\partial t^{k}}\left(\delta V_{\mathbf{y}}(t)\right)\right|_{t=0}=C_{k}(\mathbf{y})=0
$$

for $k<K$. It is important to note that $\mathcal{M}_{\mathbf{y}}(0) \neq 0$. This follows since

$$
\left[H\left(t_{*}\right), n_{\mathbf{r}}\left(t_{*}\right)\right] \neq 0
$$

for $t_{*} \neq T$, and so the eigenstates of $H\left(t_{*}\right)$ (specifically, its ground-state $\left|\psi_{0}\right\rangle$ ) cannot be fermion number eigenstates. This ensures that the ground-state expectation value

$$
\mathcal{M}_{\mathbf{y}}(0)=\left\langle\psi_{0}\left|\left(a_{\mathbf{y}}^{\dagger} Q_{\mathbf{y}}+Q_{\mathbf{y}}^{\dagger} a_{\mathbf{y}}\right)\right| \psi_{0}\right\rangle \neq 0
$$

for $t_{*} \neq T$. It follows from the continuity equation for the fermion current that

$$
\frac{\partial}{\partial t}\left(n_{\mathbf{r}}(t)-n_{\mathbf{r}}^{\prime}(t)\right)=-\sum_{k=1,2} \Delta_{k}\left\{\delta j_{\mathbf{r}, k}(t)\right\} .
$$

Taking $\mathrm{K}$ time-derivatives of this equation, evaluating the result at $t=0$, and using Eq. (17) gives

$$
\left.\frac{\partial^{K+2}}{\partial t^{K+2}}\left(n_{\mathbf{r}}(t)-n_{\mathbf{r}}^{\prime}(t)\right)\right|_{t=0}=-C_{K}(\mathbf{r}) \mathcal{M}_{\mathbf{r}}(0) \neq 0,
$$

where we have used the equation of motion for $G_{\mathbf{r}, \mathbf{y}}$. Equation (18) indicates that $n_{\mathbf{r}}(t)$ cannot equal $n_{\mathbf{r}}^{\prime}(t)$ since it insures that they will be different at $t=0^{+}$, and so cannot be the same function. This proves the Runge-Gross theorem for the instantaneous MAXCUT dynamics. 
We have just seen that when potentials $V_{\mathbf{r}}(t)$ and $V_{\mathbf{r}}^{\prime}(t)$ differ by a time-dependent function $C(t)$, they give rise to the same $\mathrm{SOF} n_{\mathbf{r}}(t)$. However, the wavefunctions produced by these potentials from the same initial state will differ by a time-dependent phase factor. For our purposes, it is important to note that this extra phase factor cancels out when calculating the expectation value of an operator. In particular, it will cancel out when calculating the instantaneous energy eigenvalues $E_{n}(t)=\left\langle E_{n}(t)|H(t)| E_{n}(t)\right\rangle$. As a result, this phase factor will not affect our calculation of the minimum energy gap in Section $\mathrm{V}$. Having said that, it is worth noting that this subtlety is not expected to cause difficulties in practice since the probe potential $V_{\mathbf{r}}(t)$ is assumed to be under the direct control of the experimenter, and so the precise form of $V_{\mathbf{r}}(t)$ is known. When an experimentalist says a sinusoidal probe potential has been applied, this means $V_{\mathbf{r}}(t)=V_{\mathbf{r}} \sin \omega t$; it does not mean $V_{\mathbf{r}}(t)=V_{\mathbf{r}} \sin \omega t+C(t)$. Thus in a well-designed experiment $C(t)=0$.

The Kohn-Sham (KS) system of non-interacting fermions can also be introduced in TDDFT 15 . We must still assume that the interacting SOF $n_{\mathbf{r}}(t)$ can be obtained from the SOF of the non-interacting KS fermions moving in the external potential $v_{\mathbf{r}}^{k s}(t)$. The potentials $v_{\mathbf{r}}^{k s}(t)$ and $v_{\mathbf{r}}(t)$ are related via $\left(t_{*} \neq 0\right)$

$$
v_{\mathbf{r}}^{k s}(t)=v_{\mathbf{r}}(t)+\left(\frac{T}{t_{*}}\right) v_{x c}[n(t)](\mathbf{r})
$$

though Eq. (19) is to be thought of as defining the time-dependent exchange-correlation potential $v_{x c}[n(t)](\mathbf{r})$.

\section{MINIMUM GAP}

A problem of longstanding treachery in GS-DFT is the calculation of the excitation energies of a fermion system. TD-DFT was able to find these energies by determining the system's frequency-dependent linear response, and relating the excitation energies to poles appearing in that response. The arguments used $\underline{31}$ are quite general, and can be easily adapted to determine the energy gap for the instantaneous MAXCUT dynamics.

Previously, we considered an external potential that becomes time-varying for $t \geq 0$. Our interest is in the interacting fermion linear response, and so we assume that the total potential has the form

$$
v_{\mathbf{r}}^{\text {tot }}(t)=v_{\mathbf{r}}+v_{\mathbf{r}}^{1}(t),
$$


with $v_{\mathbf{r}}^{1}(t)$ a suitably small time-varying perturbation. The probe potential $v_{\mathbf{r}}^{1}(t)$ generates a first-order response $n_{\mathbf{r}}^{1}(t)$ in the SOF:

$$
n_{\mathbf{r}}^{\text {tot }}(t)=n_{\mathbf{r}}^{g}+n_{\mathbf{r}}^{1}(t)
$$

The susceptibility $\chi_{\mathbf{r}, \mathbf{r}^{\prime}}\left(t-t^{\prime}\right)$ connects the first-order probe potential to the SOF response. The total potential $v_{\mathbf{r}}^{\text {tot }}(t)$ is related to the KS potential $v_{\mathbf{r}}^{k s}(t)$ through Eq. (19), and by assumption, the SOF for both the interacting and KS fermions is the same. This allows the time-Fourier transform of the SOF response $n_{\mathbf{r}}^{1}(\omega)$ to be determined from the time-Fourier transforms of the KS susceptibility $\chi_{\mathbf{r}, \mathbf{r}^{\prime}}^{k s}(\omega)$, the exchange-correlation kernel $f_{x c}\left[n^{g}\right]\left(\mathbf{r}, \mathbf{r}^{\prime} ; \omega\right)$, and the probe potential $v_{\mathbf{r}}^{1}(\omega)$ :

$$
\begin{array}{r}
\sum_{\mathbf{y}^{\prime}}\left\{\delta_{\mathbf{r}, \mathbf{y}^{\prime}}-\sum_{\mathbf{r}^{\prime}} \chi_{\mathbf{r}, \mathbf{r}^{\prime}}^{k s}(\omega) f_{x c}\left[n^{g}\right]\left(\mathbf{r}^{\prime}, \mathbf{y}^{\prime} ; \omega\right)\right\} n_{\mathbf{y}^{\prime}}^{1}(\omega) \\
=\sum_{\mathbf{r}^{\prime}} \chi_{\mathbf{r}, \mathbf{r}^{\prime}}^{k s}(\omega) v_{\mathbf{r}^{\prime}}^{1}(\omega) .
\end{array}
$$

The KS susceptibility $\underline{\underline{16}}$ depends on the KS static unperturbed orbitals $\phi_{\mathbf{r}}^{j}$; and the corresponding energy eigenvalues $\varepsilon_{j}$ and orbital occupation numbers $f_{j}$ :

$$
\chi_{\mathbf{r}, \mathbf{r}^{\prime}}^{k s}(\omega)=\sum_{j, k}\left(f_{k}-f_{j}\right) \frac{\phi_{j}(\mathbf{r}) \bar{\phi}_{k}(\mathbf{r}) \bar{\phi}_{j}\left(\mathbf{r}^{\prime}\right) \phi_{k}\left(\mathbf{r}^{\prime}\right)}{\omega-\left(\varepsilon_{j}-\varepsilon_{k}\right)+i \eta} .
$$

The exchange-correlation kernel $f_{x c}\left[n^{g}\right]$ incorporates all many-body effects into the linear response dynamics, and is related to the exchange-correlation potential $v_{x c}\left[n^{g}\right]$ through a functional derivative:

$$
f_{x c}\left[n^{g}\right]=\frac{\delta v_{x c}\left[n^{g}\right]}{\delta n^{g}} .
$$

In general, the interacting fermion excitation energies

$$
\Omega_{j k}=E_{j}-E_{k}
$$

differ from the KS excitation energies

$$
\omega_{j k}=\varepsilon_{j}-\varepsilon_{k}
$$

The RHS of Eq. (20) remains finite as $\omega \rightarrow \Omega_{j k}$, while the first-order SOF response $n_{\mathbf{y}^{\prime}}^{1}(\omega)$ has a pole at each $\Omega_{j k}$. Thus the operator on the LHS acting on $n_{\mathbf{y}^{\prime}}^{1}(\omega)$ cannot be invertible. Otherwise, its inverse could be applied to both sides of Eq. (20) with the result that the RHS 
would remain finite as $\omega \rightarrow \Omega_{j k}$, while the LHS would diverge. To avoid this inconsistency, the operator must have a zero eigenvalue as $\omega \rightarrow \Omega_{j k}$. Following Ref. 31 , one is led to the following eigenvalue problem:

$$
\sum_{k^{\prime}, j^{\prime}} \frac{M_{k j ; k^{\prime} j^{\prime}}(\omega)}{\omega-\omega_{j^{\prime} k^{\prime}}+i \eta} \xi_{k^{\prime} j^{\prime}}(\omega)=\lambda(\omega) \xi_{k j}(\omega),
$$

where, writing

$$
\alpha_{k^{\prime} j^{\prime}}=f_{k^{\prime}}-f_{j^{\prime}}
$$

and

$$
\Phi_{\mathbf{r}}^{k j}=\bar{\phi}_{k}(\mathbf{r}) \phi_{j}(\mathbf{r})
$$

we have

$$
M_{k^{\prime} j^{\prime} ; k j}(\omega)=\alpha_{k^{\prime} j^{\prime}} \sum_{\mathbf{r}^{\prime}, \mathbf{y}^{\prime}} \Phi_{\mathbf{r}^{\prime}}^{k j}\left\{f_{x c}\left[n^{g}\right]\left(\mathbf{r}^{\prime}, \mathbf{y}^{\prime} ; \omega\right)\right\} \Phi_{\mathbf{y}^{\prime}}^{k^{\prime} j^{\prime}}(\omega) .
$$

It can be shown that $\lambda\left(\Omega_{j k}\right)=1$.

At this point in the argument, it proves necessary to introduce some form of approximation to proceed further. In the single-pole approximation ${ }^{31}$ the KS poles are assumed to be well-separated so that we can focus on a particular KS excitation energy $\omega_{j k}=\omega_{*}$. The eigenvectors $\xi_{k^{\prime} j^{\prime}}(\omega)$ and the matrix operator $M_{k j ; k^{\prime} j^{\prime}}(\omega)$ are finite at $\omega_{*}$, while the eigenvalue $\lambda(\omega)$ must have a pole there to match the pole on the LHS of Eq. (22):

$$
\lambda(\omega)=\frac{A\left(\omega_{*}\right)}{\left(\omega-\omega_{*}\right)}+\mathcal{O}(1) .
$$

Let $\omega_{*}$ be $d$-fold degenerate: $\omega_{k_{1} j_{1}}, \cdots, \omega_{k_{d} j_{d}}=\omega_{*}$. Matching singularities in Eq. (22) gives

$$
\sum_{l=1}^{d} M_{k_{i} j_{i} ; k_{l}^{\prime} j_{l}^{\prime}}\left(\omega_{*}\right) \xi_{k_{l}^{\prime} j_{l}^{\prime}}^{n}=A^{n}\left(\omega_{*}\right) \xi_{k_{i} j_{i}}^{n}\left(\omega_{*}\right)
$$

where $i, n=1, \ldots, d$. For our purposes, the eigenvalues $A^{n}\left(\omega_{*}\right)$ are of primary interest and are found from Eq. (23) . From each $A^{n}\left(\omega_{*}\right)$, we find

$$
\lambda^{n}(\omega)=\frac{A^{n}\left(\omega_{*}\right)}{\left(\omega-\omega_{*}\right)} .
$$

Since $\lambda^{n}\left(\Omega_{j k}\right)=1$, it follows that the sum of $\lambda^{n}\left(\Omega_{j k}\right)$ and its complex conjugate is 2 . Plugging into this sum the singular expressions for $\lambda^{n}\left(\Omega_{j k}\right)$ and that of its complex conjugate, and solving for $\Omega_{j k}^{n}$ gives

$$
\Omega_{j k}^{n}=\omega_{*}+\operatorname{Re}\left[A^{n}\left(\omega_{*}\right)\right] .
$$


Interactions will thus generally split the $\omega_{*}$-degeneracy. Now let

$$
\delta E=\min _{n} \operatorname{Re}\left[A^{n}\left(\omega_{*}\right)\right]
$$

and

$$
\bar{\Omega}_{j k}=\min _{n} \Omega_{j k}^{n} .
$$

Our expression for $\Omega_{j k}^{n}$ then gives

$$
\bar{\Omega}_{j k}=\omega_{*}+\delta E .
$$

In the context of the QAE algorithm, our interest is the energy gap

$$
\Delta\left(t_{*}\right)=E_{1}\left(t_{*}\right)-E_{0}\left(t_{*}\right)
$$

separating the instantaneous ground and first-excited states. In this case, our expression for $\bar{\Omega}_{j k}$ gives

$$
\Delta\left(t_{*}\right)=\left[\varepsilon_{1}\left(t_{*}\right)-\varepsilon_{0}\left(t_{*}\right)\right]+\delta E\left(t_{*}\right) .
$$

To obtain the minimum gap $\Delta$ for QAE numerically, one picks a sufficiently large number of $t_{*} \in(0, T)$; solves for $\Delta\left(t_{*}\right)$ using the KS system associated with $H\left(t_{*}\right)$ to evaluate the RHS of Eq. (24); then uses the minimum of the resulting set of $\Delta\left(t_{*}\right)$ to upper bound $\Delta$. Because the KS dynamics is non-interacting, it has been possible to treat KS systems with $N \sim 10^{3} \mathrm{KS}$ fermions $\underline{17}, \underline{18}, \underline{19}$. This would allow the evaluation of the minimum gap $\Delta(N)$ for the QAE algorithm for $N \sim 10^{3}$.

\section{DISCUSSION}

As with all KS calculations, the minimum gap calculation requires an approximation for the exchange-correlation energy functional $\xi_{x c}[n]$. Note that, because the qubits in a quantum register must be located at fixed positions for the register to function properly, the associated JW fermions are distinguishable since they are each pinned to a specific lattice site. Consequently, anti-symmetrization of the fermion wavefunction is not required, with the result that the exchange energy vanishes in the MAXCUT dynamics. The exchangecorrelation energy functional $\xi_{x c}[n]$ is then determined solely by the correlation energy which can be calculated using the methods of Ref. 32. Parametrization of these results yields 
analytical expressions for the correlation energy per particle which, upon differentiation, give $v_{x c}[n]$ and $f_{x c}[n]$. Replacing $n \rightarrow n_{\mathbf{r}}$ in $\xi_{x c}[n]$ gives the local density approximation (LDA) for GS-DFT; while $n \rightarrow n_{\mathbf{r}}(t)$ gives the adiabatic local density approximation (ALDA) for TDDFT. These simple approximations have proven to be remarkably successful, and provide a good starting point for the minimum gap calculation. Self-interaction corrections to $\xi_{x c}[n]$ are not necessary since the two-fermion interaction [see Eq. (10)] has no self-interaction terms. Finally, because the fermions are pinned, it will be necessary to test the gap for sensitivity to derivative discontinuities ${ }^{33}$ in $\xi_{c}[n]$.

\section{Acknowledgments}

We thank A. Satanin for stimulating conversations. F. Nori gratefully acknowledges partial support from the NSA, LPS, ARO, and NSF grant No. EIA-0130383; and F. Gaitan thanks T. Howell III for continued support.

1 E. Farhi et al., Science 292, 472 (2001).

2 F. Gaitan, Int. J. Quantum Info. 4, 843 (2006); F. Gaitan, to appear in Complexity.

3 E. Farhi et al., available at arXiv.org/quant-ph/0001106.

4 S. Ashhab, J.R. Johansson, and F. Nori, Phys. Rev. A 74, 052330 (2006).

5 A.M. Zagoskin, S. Savelev, and F. Nori, Phys. Rev. Lett. 98, 120503 (2007).

6 M.R. Garey and D.S. Johnson, Computers and Intractability (W.H. Freeman, New York, 1979).

7 T. Hogg, Phys. Rev. A 67, 022314 (2003).

8 A. Young, S. Knysh, and V.N. Smelyanskiy, Phys. Rev. Lett. 101, 170503 (2008).

9 I.M. Buluta and F. Nori, preprint.

10 R. Dreizler and E. Gross, Density Functional Theory (Springer-Verlag, New York, 1990).

11 R.G. Parr and W. Yang, Density-Functional Theory of Atoms and Molecules (Oxford, New York, 1989).

12 M.A.L. Marques et al., Time-Dependent Density Functional Theory (Springer, Berlin Heidelberg, 2006).

13 P. Hohenberg and W. Kohn, Phys. Rev. 136, B864 (1964). 
14 W. Kohn and L.J. Sham, Phys. Rev. 140, A1133 (1965).

15 E. Runge and E. Gross, Phys. Rev. Lett. 52, 997 (1984).

16 M. Marques and E. Gross, Annu. Rev. Phys. Chem. 55, 427 (2004).

17 F. Shimojo, R.K. Kalia, A. Nakano, and P. Vashishta, Phys. Rev. B 77095103 (2008).

18 H. Jiang, H.U. Baranger, and W. Yang, Phys. Rev. B 68, 165337 (2003).

19 D. Sanchez-Portal, P. Ordejon, E. Artacho, and J.M. Soler, Int. J. Quantum Chem. 65, 453 (1997).

20 G. Chen et al., Quantum Computing Devices (CRC Press, Boca Raton, FL 2007).

21 J.Q. You and F. Nori, Phys. Today 58 (11), 42 (2005).

22 M. Steffen et al., Phys. Rev. Lett. 90, 067903 (2003).

23 E. Fradkin, Phys. Rev. Lett. 63, 322 (1989); Field Theories of Condensed Matter Systems (Addison-Wesley, New York 1991).

24 C.D. Batista and G. Ortiz, Phys. Rev. Lett. 86, 1082 (2001).

25 The component $A_{0}(\mathbf{r})$ remains classical since its canonically conjugate momentum vanishes: $\Pi_{0}=0$. Its equation of motion (Gauss' law) becomes $\delta L / \delta A_{0}=0$ which is imposed as a constraint on physical states. See for example, R. Jackiw in Current Algebra and Anomalies, edited by S.B. Treiman et al., (Princeton University Press, Princeton, NJ, 1985).

26 W. Kohn, in Highlights of Condensed Matter Theory, edited by F. Bassani et al. (North-Holland, Amsterdam, 1985).

27 R.M. Driezler and E.K.U. Gross, Density Functional Theory (Springer, Berlin, 1990).

28 M. Levy, Proc. Natl. Acad. Sci. U. S. A. 76, 6062 (1979).

29 V.V. Franca and K. Capelle, Phys. Rev. Lett. 100, 070403 (2008); Phys. Rev. A 77, 062324 (2008).

30 L.-A. Wu, M.S. Sarandy, D.A. Lidar, and L.J. Sham, Phys. Rev. A 74, 052335 (2006).

31 M. Petersilka, U.J. Gossmann, and E.K.U. Gross, Phys. Rev. Lett. 76, 1212 (1996).

32 D. Ceperley and B. Adler, Phys. Rev. Lett. 45, 566 (1980).

33 J. Perdew and M. Levy, Phys. Rev. Lett. 51, 1884 (1983); L. Sham and M. Schlüter, Phys. Rev. Lett. 51, 1888 (1983). 